\title{
Emissão luminescente no titanato de cálcio dopado com íons de terras-raras
}

\section{(Luminescent emission in calcium titanate doped with rare-earth ions)}

\author{
S. C. Pereira', M. M. Paranhos ${ }^{1}$, A. C. Eduardo ${ }^{1}$, M. A. B. Santos ${ }^{2}$, M. S. Li ${ }^{3}$, \\ E. Longo ${ }^{4}$, F. V. Motta ${ }^{5}$ A. T. de Figueiredo ${ }^{1}$ \\ ${ }^{1}$ Programa de pós-graduação em Química - UFG/CAC - Catalão, GO \\ ${ }^{2}$ Curso de Química - UFG/CAC - Catalão, GO \\ ${ }^{3}$ IFSC, USP, S. Carlos, SP \\ ${ }^{4}$ LIEC - Instituto de Química, UNESP, Araraquara, SP \\ ${ }^{5}$ DEMat-CT - UFRN - Natal, RN \\ *sandrinhadcp@yahoo.com.br
}

\begin{abstract}
Resumo
Através do método dos precursores poliméricos foram sintetizadas amostras de $\mathrm{Ca}_{0,98} \mathrm{Sr}_{0,01} \mathrm{X}_{0,01} \operatorname{TiO}_{3}\left(\mathrm{X}=\mathrm{Eu}^{3+}, \mathrm{Sm}^{3+} \mathrm{ou}^{3+}\right)$, que submetidas a diferentes tratamentos térmicos foram obtidas com níveis de ordem-desordem estrutural distintos. Os materiais sintetizados foram caracterizados por difração de raios X, confirmando a obtenção de um material homogêneo com estrutura perovsquita do tipo ortorrômbica. Essa caracterização permitiu ainda calcular o tamanho médio de cristalito das amostras, que varia em função do íon terra-rara presente. Os resultados da emissão fotoluminescente confirmaram a presença desse fenômeno óptico à temperatura ambiente e sua relação com o nível de ordem-desordem estrutural do sistema. A emissão fotoluminescente mais intensa ocorre nas amostras tratadas termicamente a $500^{\circ} \mathrm{C}$ (independente do íon dopante), a variação do dopante influencia na intensidade da emissão devido à transferência de carga entre a matriz hospedeira e o íon terra-rara emissor, onde a amostra dopada com íon $\mathrm{Pr}^{3+}$ apresenta transferência de carga mais eficiente e consequentemente maior intensidade na emissão fotoluminescente. Outro fator que favorece a propriedade óptica das amostras é a compensação de carga, pois contribui para o aumento da desordem estrutural devido à formação de vacâncias do íon $\mathrm{Ca}^{2+}$.

Palavras-chave: perovsquita, titanato de cálcio, terras-raras, fotoluminescência.
\end{abstract}

\begin{abstract}

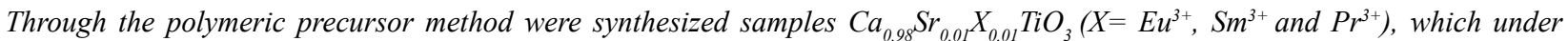
different heat treatments were obtained with levels of distinct structural order-disorder. The synthesized materials were characterized by X-ray diffraction, confirming the obtaining of ahomogeneous material with perovskite-type orthorhombic structure. This characterization allowed evaluating the average crystallite size of the samples that varies depending on the rare-earth ion. The results of photoluminescence emission confirmed the presence of this optical phenomenon at room temperature and its relationship to the level of order-disorder structural system. The photoluminescence emission is more intense in samples annealed at $500{ }^{\circ} \mathrm{C}$ (independent on the dopant ion), the variation of the dopant influence on the emission intensity due to charge transfer between the host and emitting rare-earth ion, where the doped with $\mathrm{Pr}^{3+}$ ion has charge transfer more efficient and hence more intense emission in the photoluminescence. Another factor that favors the optical property of the samples is the charge compensation, as it contributes to the increase in structural disorder due to formation of $\mathrm{Ca}^{2+}$ vacancies.
\end{abstract}

Keywords: perovskite, calcium titanate, rare-earth, photoluminescence.

\section{INTRODUÇÃO}

Nos últimos anos, óxidos do tipo perovsquita dopados com íons terras raras tem atraído grande interesse, principalmente na área da fotoluminescência. A propriedade fotoluminescente (FL) sempre despertou o interesse da comunidade científica, principalmente a partir da descoberta da fotoluminescência na temperatura ambiente, o que favorece as aplicações tecnológicas como em diodos emissores de luz (LED), lasers na região da luz visível e componentes de fibra ópticas [1-5]. A fim de entender e melhorar a emissão luminescente, vários estudos tem sido voltados para esses materiais através de mudanças, tais como: a variação do íon dopante [6-10], a concentração do íon dopante [11-13], a matriz hospedeira [8, 14-19], a presença de compensadores de carga $[19,20]$ e o uso de diferentes métodos de síntese [14, 20-24].

Entre as perovsquitas dedicadas a aplicações ópticas, o titanato de cálcio dopado com $\mathrm{Pr}^{3+}\left(\mathrm{CaTiO}_{3}: \mathrm{Pr}^{3+}\right)$ é um material que apresenta interesse especial, uma vez que esse fósforo exibe catodoluminescência vermelha com coordenadas muito próximas do "vermelho ideal". Essa emissão é muito intensa e nítida em $610 \mathrm{~nm}$, podendo ser atribuída à transição ${ }^{1} \mathrm{D}_{2} \rightarrow{ }^{3} \mathrm{H}_{4}$ no íon praseodímio $[1,10,19,25]$. 
Vários trabalhos explicam as condições que favorecem a emissão FL em materiais. Alguns autores atribuem a emissão FL a desvios de estequiometria [26, 27] e outros a presença de impurezas [28, 29]. No entanto, existe um consenso geral na literatura de que a emissão $\mathrm{FL}$ está relacionada à ordem-desordem estrutural presente nos materiais [30-32]. A potencialização da FL também ocorre pela compensação de carga, que ocorre pela formação de defeitos intrínsecos, tais como, vacâncias de íons na rede cristalina [26, 33-36].

O método de síntese pode afetar a emissão luminescente do material. Dentre esses métodos convém destacar o método convencional de reação do estado sólido para a preparação de fósforos (requer altas temperaturas de calcinação) [37, $38]$, o método sol-gel [28, 39], o método hidrotérmico [25, 40], dentre outros. Contudo, o Método dos Precursores Poliméricos, utilizado nesse trabalho, é simples, rápido e possibilita um alto controle estequiométrico [41-43].

Neste trabalho, será apresentada a síntese de materiais baseados na estrutura do titanato de cálcio. Será promovida desordem estrutural através de distorções locais associadas com a substituição de cálcio por estrôncio. Com o intuito de estudar a emissão fotoluminescente de íons terras-raras na matriz hospedeira $\mathrm{Ca}_{0,99} \mathrm{Sr}_{0,01} \mathrm{TiO}_{3}$ esse material será também dopado com os íons $\mathrm{Eu}^{3+}, \mathrm{Pr}^{3+}$ e $\mathrm{Sm}^{3+}$. Todos os materiais serão obtidos pelo Método dos Precursores Poliméricos. A emissão FL apresentada por estes materiais será discutida em função dos diferentes dopantes e da ordem estrutural.

\section{EXPERIMENTAL}

Os pós de $\mathrm{Ca}_{0,98} \mathrm{Sr}_{0,01} \mathrm{X}_{0,01} \mathrm{TiO}_{3}$ dopados com $1 \%$ de terrasraras, $\mathrm{CST}: \mathrm{X}\left(\mathrm{X}=\mathrm{Eu}^{3+}, \mathrm{Sm}^{3+}\right.$ ou $\left.\mathrm{Pr}^{3+}\right)$, foram obtidos pelo método dos precursores poliméricos (MPP). No processo de síntese, o citrato de titânio foi obtido pela dissolução do isopropóxido de titânio (IV) em solução aquosa de ácido cítrico, sob agitação e temperatura próximo a $70{ }^{\circ} \mathrm{C}$. Após a homogeneização da solução de citrato de $\mathrm{Ti}, \mathrm{CaCO}_{3}$ foi adicionado lentamente, e posteriormente foi adicionado $\mathrm{SrCO}_{3}$. Em seguida foi adicionado o precursor do íon terra-rara de interesse, $\operatorname{Pr}\left(\mathrm{NO}_{3}\right)_{3}, \mathrm{Eu}\left(\mathrm{NO}_{3}\right)_{3}$ ou $\mathrm{Sm}_{2} \mathrm{O}_{3}$. O último foi previamente aberto em ácido nítrico. A fim de promover a polimerização do citrato, através da reação de poliesterificação, foi adicionado etilenoglicol e mantido em agitação. A razão molar entre o ácido cítrico e o etilenoglicol foi fixada como 60/40 (razão em massa). A resina polimérica obtida foi calcinada a $300^{\circ} \mathrm{C}$ por $2 \mathrm{~h}$ para obtenção do "puff". Esse foi desaglomerado e pré-calcinado a $300{ }^{\circ} \mathrm{C}$ por $20 \mathrm{~h}$ para eliminação completa de matéria orgânica proveniente do método de síntese. O material livre de matéria orgânica foi submetido a tratamento térmico a 400, 450, 500, 550, 600 e $700{ }^{\circ} \mathrm{C}$ por $2 \mathrm{~h}$.

As amostras de CST:X foram caracterizadas estruturalmente por difração de raios $\mathrm{X}$ (DRX), os difratogramas foram obtidos em um difratômetro XRD 6100 Shimadzu, utilizando a radiação $\mathrm{CuK} \alpha$ do cobre $(\lambda=1,5418$ $\AA$ ). Os difratogramas foram obtidos com $2 \theta$ variando de 5 a $105^{\circ} \mathrm{e}$ foram comparados com arquivos JCPDS, pelo método computacional, com base no arquivo 42-423 do ICDD do $\mathrm{CaTiO}_{3}$. As medidas de emissão $\mathrm{FL}$ foram realizadas com comprimento de onda de excitação de $350,7 \mathrm{~nm}$ obtido de um laser com íons de kriptônio (Coherent Innova), com uma potência de saída do laser de $200 \mathrm{~mW}$. O monocromador utilizado foi um Thermal Jarrel-Ash Monospec 27. Foi utilizada uma fotomultiplicadora Hamamatsu R446 acoplada a um sistema de aquisição composto de um "lock-in" SR530 controlado por um microcomputador. Todas as medidas foram feitas a temperatura ambiente.

\section{RESULTADOS E DISCUSSÃO}

O padrão de difração de todas as amostras preparadas é mostrado na Fig. 1. Independentemente do dopante utilizado, foi detectado apenas picos relacionados à estrutura ortorrômbica do tipo perovsquita do $\mathrm{CaTiO}_{3}$, com grupo espacial Pbnm, acima de cada pico no difratograma encontra-se a identificação dos planos cristalinos. Todos os picos de difração foram indexados com base no arquivo ICDD 42-423. Picos de difração adicionais referentes aos precursores ou fases adicionais não são observados, portanto todas as amostras podem ser consideradas monofásicas. Em adição, não foram observadas alterações na estrutura referentes à presença do íon $\mathrm{Sr}^{2+}$ diferentemente do que é observado quando a concentração do dopante estrôncio é alta no sistema $\mathrm{Ca}_{1-x} \mathrm{Sr}_{\mathrm{x}} \mathrm{TiO}_{3}$ [44-47].

A técnica de difração de raios $\mathrm{X}$, além de ser utilizada para avaliar a obtenção da fase, também pode ser para calcular o tamanho médio dos cristalitos por meio da equação de Scherrer [48]:

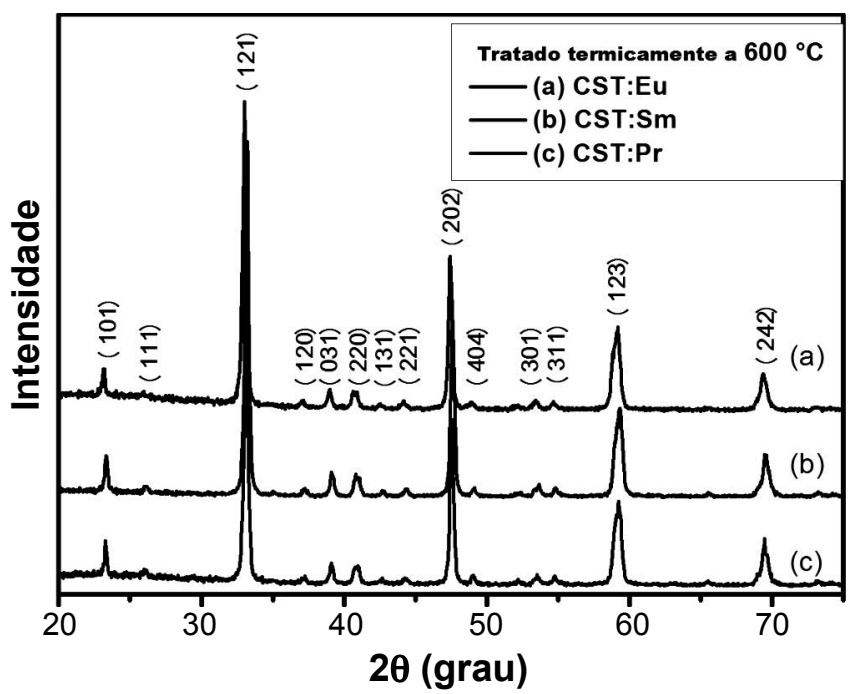

Figura 1: Padrões de difração das amostras de CST:X $(X=E u, S m$ e Pr) tratadas termicamente a $600{ }^{\circ} \mathrm{C}$.

[Figure 1: XRD patterns of samples CST: $X(X=E u, S m$, and Pr) heat treated at $600^{\circ} \mathrm{C}$.]

$$
t=0,9 \lambda / B \cos \theta
$$

na qual $\mathrm{B}^{2}=\left(\mathrm{B}_{\mathrm{obs}}\right)^{2}-\left(\mathrm{B}_{\mathrm{p}}\right)^{2}, \lambda$ é o comprimento de onda utilizado, $\theta$ o ângulo de difração e $B$ a largura à meia altura 
Tabela I - Tamanho médio do cristalito das amostras CST:X $\left(\mathrm{X}=\mathrm{Eu}^{3+}, \mathrm{Sm}^{3+}\right.$ e $\left.\mathrm{Pr}^{3+}\right)$.

[Table I - Mean crystallite size of the samples CST:X $\left(X=\mathrm{Eu}^{3+}, \mathrm{Sm}^{3+}\right.$ and $\left.\left.\mathrm{Pr}^{3+}\right).\right]$

\begin{tabular}{cc}
\hline $\begin{array}{c}\text { Amostras tratadas } \\
\text { termicamente a } 700{ }^{\circ} \mathrm{C}\end{array}$ & $\begin{array}{c}\text { Tamanho médio de } \\
\text { cristalito (nm) }\end{array}$ \\
\hline $\mathrm{Ca}_{0,98} \mathrm{Sr}_{0,01} \mathrm{TiO}_{3}: \mathrm{Sm}_{0,01}$ & 48,59 \\
$\mathrm{Ca}_{0,98} \mathrm{Sr}_{0,01} \mathrm{TiO}_{3}: \mathrm{Pr}_{0,01}$ & 49,59 \\
$\mathrm{Ca}_{0,98} \mathrm{Sr}_{0,01} \mathrm{TiO}_{3}: \mathrm{Eu}_{0,01}$ & 50,06 \\
\hline
\end{tabular}

do pico de difração. $B_{o b s}$ é a largura à meia altura do resultado experimental e $B_{p}$ é o resultado de um padrão monocristalino externo analisado nas mesmas condições da amostra. Para se realizar tal cálculo, o pico de difração foi adequado a uma função do tipo gaussiana, via métodos computacionais, para se medir a largura a meia altura do mesmo. A Tabela I mostra os resultados obtidos para as amostras CST:X $\left(\mathrm{X}=\mathrm{Eu}^{3+}\right.$, $\mathrm{Sm}^{3+}$ e $\mathrm{Pr}^{3+}$ ) tratadas termicamente a $700{ }^{\circ} \mathrm{C}$ Os resultados mostram que o tamanho médio do cristalito é influenciado pelo íon terra-rara presente.

Embora a matriz hospedeira de $\mathrm{Ca}_{0,98} \mathrm{Sr}_{0,01} \mathrm{X}_{0,01} \mathrm{TiO}_{3}$ seja mantida em todos os materiais obtidos, e com isso os padrões de difração observados para os materiais com diferentes dopantes sejam idênticos, a emissão FL mostrase completamente distinta quanto a aspecto e intensidade. A Fig. 2 ilustra os espectros de emissão FL das amostras tratadas termicamente a $600{ }^{\circ} \mathrm{C}$, obtidas com comprimento de onda de excitação de $350,7 \mathrm{~nm}$ para os diferentes materiais obtidos.

É possível observar apenas picos intensos de emissão, os quais são referentes às transições internas da camada semi preenchida $f$ dos íons terras-raras presentes na matriz hospedeira $\mathrm{Ca}_{0,98} \mathrm{Sr}_{0,01} \mathrm{X}_{0,01} \mathrm{TiO}_{3}\left(\mathrm{Eu}^{3+}-4 f^{6}, \mathrm{Sm}-4 f^{5}\right.$ e $\mathrm{Pr}$ $-4 f^{2}$ ). A emissão FL é intrínseca a cada íon. No espectro do CST:Eu os picos observados são devido as transições do estado excitado ${ }^{5} \mathrm{D}_{0}$ para o estado fundamental ${ }^{7} \mathrm{~F}_{\mathrm{J}}(\mathrm{J}=0,1-4)$. Os picos em 557, 594, 616, 655 e $696 \mathrm{~nm}$ são referentes às transições ${ }^{5} \mathrm{D}_{0} \rightarrow{ }^{7} \mathrm{~F}_{\mathrm{J}}(\mathrm{J}=0,1-4)$ [25]. Os picos observados no espectro do CST:Sm são correspondentes às transições ${ }^{4} \mathrm{G}_{5 / 2} \rightarrow{ }^{6} \mathrm{H}_{\mathrm{J}}(\mathrm{J}=5 / 2,7 / 2,9 / 2$ e $11 / 2)$, onde ${ }^{4} \mathrm{G}_{5 / 2}$ é o estado fundamental e ${ }^{6} \mathrm{H}_{\mathrm{J}}$ são os estados excitados do íon $\mathrm{Sm}^{3+}[49$, 50]. O pico observado em $617 \mathrm{~nm}$ no espectro do CST:Pr é referente a transição do estado fundamental ${ }^{1} \mathrm{D}_{2}$ para o estado excitado ${ }^{3} \mathrm{H}_{4}[19,51]$. A emissão $\mathrm{FL}$ do íon praseodímio no CST:Pr é o que apresenta a maior intensidade. Tal fato está relacionado à maior eficiência na transferência de carga da matriz hospedeira para o íon terra-rara emissor, $\mathrm{Pr}^{3+}$. Essa intensa emissão $\mathrm{FL}$ do $\mathrm{Pr}^{3+}$ é reportada para CT dopado com esse íon [1, 10, 52]. A Fig. 3 apresenta a emissão FL do CST:Sm tratado termicamente a diferentes temperaturas. Esse material foi escolhido para este estudo porque para o material tratado termicamente a temperatura inferior à necessária para cristalização apresenta mais nitidamente picos do íon terra-rara e uma banda larga referente ao
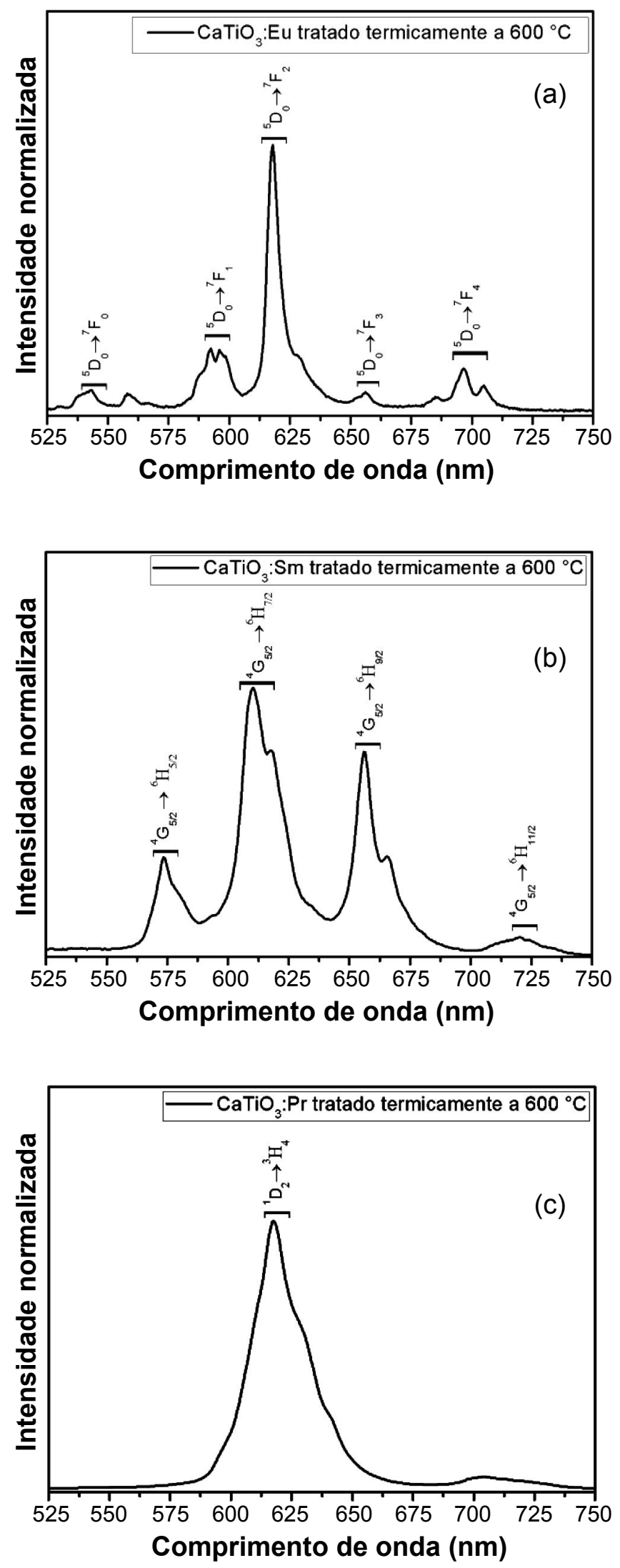

Figura 2: Espectros de FL das amostras CST:X, X=Eu, Sm ou Pr tratadas termicamente a $600{ }^{\circ} \mathrm{C}$.

[Figure 2: FL spectra of the samples CST:X, X=Eu, Sm or Pr heat treated at $600^{\circ} \mathrm{C}$.] 


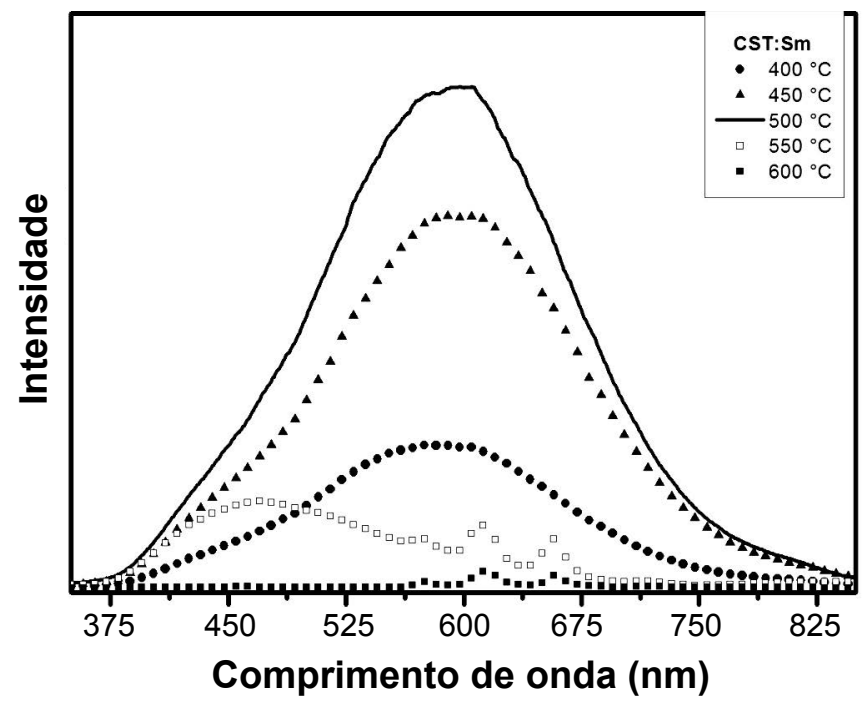

Figura 3: Espectros de emissão fotoluminescente do CST:Sm tratadas termicamente em diferentes temperaturas.

[Figure 3: Photoluminescence emission spectra of CST:Sm heat treated at different temperatures.]

material no mesmo espectro (amostra tradada termicamente a $\left.550{ }^{\circ} \mathrm{C}\right)$.

A desordem estrutural está presente quando o material é tratado termicamente abaixo de sua temperatura de cristalização, para o $\mathrm{Ca}_{0,98} \mathrm{Sr}_{0,01} \mathrm{X}_{0,01} \mathrm{TiO}_{3}$ a temperatura de cristalização é $600{ }^{\circ} \mathrm{C}$. Pode-se perceber que para materiais com desordem estrutural o espectro de emissão FL apresenta uma banda larga. O espectro de FLa temperatura ambiente para os materiais apresenta perfil típico de processos multifônicos, isto é, eles têm vários canais de relaxação que indicam um gap de larga energia. Assim, a emissão FL de banda larga consiste na soma das emissões individuais. Essas emissões surgem de uma recombinação radiativa entre elétrons presos e buracos

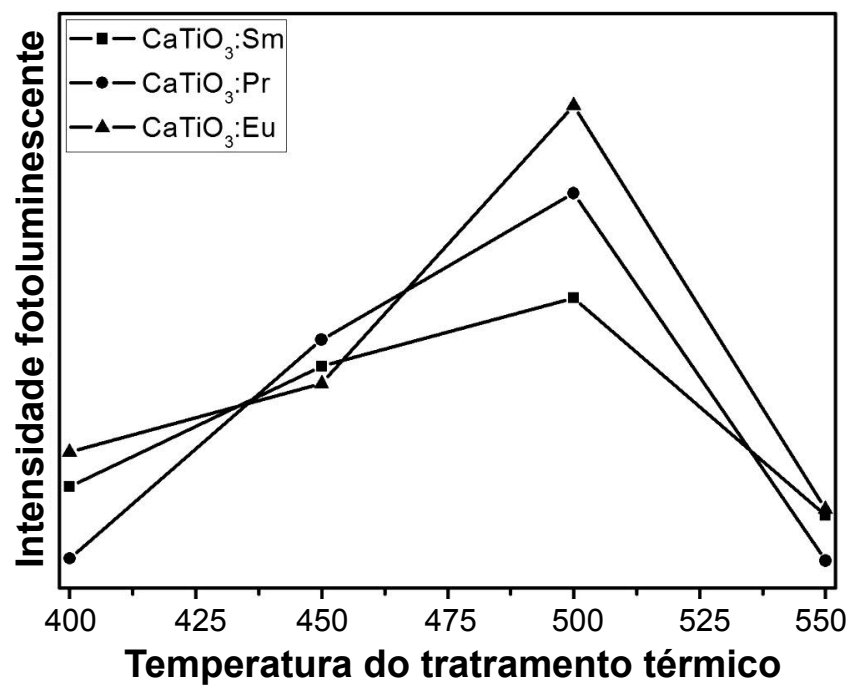

Figura 4: Comportamento fotoluminescente das amostras sintetizadas.

[Figure 4: Behavior of photoluminescence of the synthesized samples.] presos nos estados intermediarios dentro do gap. No espectro de emissão fotoluminescente da amostra calcinada a $550{ }^{\circ} \mathrm{C}$ é possível observar duas emissões luminescentes distintas, uma referente ao material (banda larga) e outra intrínseca ao íon samário (picos). É possível notar que a emissão FL do material apresenta uma banda larga (com máximo em 470 $\mathrm{nm}$ ) e estende-se de 350 a $850 \mathrm{~nm}$, enquanto a emissão do íon samário apresenta picos que podem ser notados em 567 , 611 e 657 nm. Essas emissões existem independentemente uma da outra. A interação entre elas permanece não reportada na literatura. Quando o material apresenta-se completamente ordenado estruturalmente, como é o caso do material tratado a $600{ }^{\circ} \mathrm{C}$, observam-se apenas picos referentes às transições internas do íon samário (III) $4 f^{5}$. A Fig. 4 mostra que em todos os materiais o comportamento é similar.

As reações de substituição de cátions muitas vezes não consideram a polarização de cargas ao longo da rede. Esta polarização forma defeitos eletrônicos na estrutura. A presença desses defeitos é indesejável, mesmo em baixas concentrações, pois pode diminuir a emissão fotoluminescente de muitos materiais. Este problema pode ser contornado pela compensação de cargas [36].

Para avaliar a influência da compensação de carga na emissão FL, o $\mathrm{Ca}_{0,98} \mathrm{Sr}_{0,01} \mathrm{Sm}_{\mathrm{y}} \mathrm{TiO}_{3}$ (CSST:y, com y=0,01 ou $0,007)$ foi obtido pelo método dos precursores poliméricos e a variação de suas propriedades FL foi estudada considerandose a influência da compensação de carga (Fig. 5). Quando íons trivalentes são inseridos na rede do titanato de cálcio, a substituição de cátions $\mathrm{Ca}^{2+}$ pelos cátions dopantes pode ocorrer, induzindo a formação de vacâncias de cálcio para que o desequilíbrio de cargas seja compensado. A equação abaixo, na notação de Kröger e Vink, descreve a criação das vacâncias [53]:

$$
2 \mathrm{Sm}^{3+} \underset{\mathrm{Ca}^{2+}}{\longrightarrow} 2 \mathrm{Sm}_{\mathrm{Ca}}^{\cdot}+\mathrm{V}_{\mathrm{Ca}}^{\prime \prime}+\mathrm{O}_{\mathrm{o}}^{\mathrm{x}}
$$

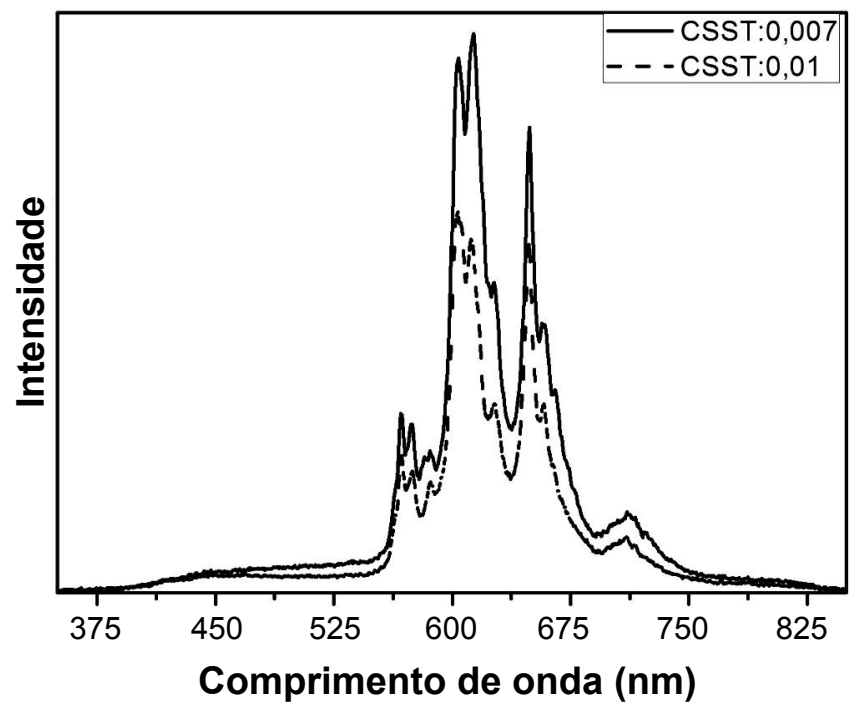

Figura 5: Espectros de $\mathrm{FL}$ do $\mathrm{Ca}_{0,98} \mathrm{Sr}_{0,01} \mathrm{Sm}_{\mathrm{y}} \mathrm{TiO}_{3}$ tratado termicamente a $500{ }^{\circ} \mathrm{C}$.

[Figure 5: PL spectra of $\mathrm{Ca}_{0,98} \mathrm{Sr}_{0,01} \mathrm{Sm}_{y} \mathrm{TiO}_{3}$ heat-treated at $500^{\circ} \mathrm{C}$.] 
na qual $2 \mathrm{Sm}_{\mathrm{Ca}}^{\circ}$ refere-se ao íons $\mathrm{Sm}^{3+}$ ocupando o sítio do $\mathrm{Ca}^{2+}, \mathrm{V}^{\prime \prime}{ }_{\mathrm{Ca}}$ representa a vacância do íon $\mathrm{Ca}^{2+} \mathrm{e} \mathrm{O}^{\mathrm{x}}{ }_{\mathrm{o}}$ representa a vacância de oxigênio neutra. Portanto a substituição do íon cálcio (II) pelo samário (III) utilizando a compensação de carga gera um buraco de íon $\mathrm{Ca}^{2+}$ na estrutura, pois para substituir 6 cargas positivas $\left(3 \mathrm{Ca}^{2+}\right)$ são necessários apenas dois íons $\mathrm{Sm}^{3+}\left(2 \mathrm{Sm}^{3+}\right)$.

Como pode ser observado na Fig. 5, o CSST:y com compensação de carga apresenta maior emissão FL. Tal fato pode ser atribuído à presença de vacâncias do íon $\mathrm{Ca}^{2+}$, que contribui para o aumento da desordem estrutural no CSST:y $(y=0,007)$. Logo, o material com a compensação de carga apresenta uma relação ordem-desordem estrutural mais adequada para a emissão FL.

\section{CONCLUSÕES}

O método dos precursores poliméricos mostrou-se eficiente na síntese de um material com estrutura perovsquita de titanato de cálcio dopado com estrôncio e diferentes íons de terras-raras. Esse método de síntese também proporciona condições favoráveis ao estudo da fotoluminescência, pois é realizado a baixas temperaturas, é de baixo custo e o material obtido é desordenado estruturalmente. Os resultados de difração de raios X mostraram a obtenção da fase de interesse e possibilitaram o cálculo do tamanho médio do cristalito, que é influenciado pela variação do íon de terra-rara. Os resultados da fotoluminescência das amostras mostraram que é necessário um nível de ordem-desordem estrutural ideal para o material exibir fotoluminescência. Esse nível ideal é alcançado quando as amostras são tratadas termicamente a $500{ }^{\circ} \mathrm{C}$ e é independente do íon terra-rara, porém a intensidade de emissão das amostras a essa temperatura são influenciadas pelo íon terra-rara, onde a presença do íon $\operatorname{Pr}^{3+}$ provoca emissão fotoluminescente mais intensa. Outro fator que influencia na intensidade FL é a compensação de carga, favorecendo a emissão devido à criação de vacâncias que contribuem para a desordem estrutural.

\section{AGRADECIMENTOS}

Os autores agradecem as agências FAPEG, CAPES e CNPq pelos recursos disponibilizados. Este trabalho utilizou infraestrutura disponibilizada pelo INCTMN e pela Rede RECAM.

\section{REFERÊNCIAS}

[1] B. Yan, K. Zhou, J. Alloy. Compd. 398 (2005) 165.

[2] B. Yan, X. Q. Su, K. Zhou, Mater. Res. Bull. 41 (2006) 134.

[3] R. Stefani, A. D. Maia, E. E. S. Teotonio, M. A. F. Monteiro, M. Felinto, H. F. Brito, J. Solid State Chem. 179 (2006) 1086.

[4] G. S. R. Raju, S. Buddhudu, J. Appl. Polym. Sci. 107 (2008) 2480.

[5] X. M. Liu, P. Y. Jia, J. Lin, G. Z. Li, J. Appl. Phys. 99
(2006).

[6] M. G. Ha, J. H. Lee, J. S. Bae, J. P. Kim, K. S. Hong, H. S. Yang, Curr. Appl. Phys. 11 (2012) 1379.

[7] M. Mohapatra, Y. P. Naik, V. Natarajan, T. K. Seshagiri, Z. Singh, S. V. Godbole, J. Lumin. 130 (2010) 2402.

[8] P. Boutinaud, L. Sarakha, R. Mahiou, P. Dorenbos, Y. Inaguma, J. Lumin. 130 (2010) 1725.

[9] K. B. Ruan, X. M. Chen, T. Liang, G. H. Wu, D. H. Bao, J. Appl. Phys. 103 (2008).

[10] S. Y. Yin, D. H. Chen, W. J. Tang, Y. H. Peng, Mater. Sci. Eng. B-Solid State Mater. Adv. Technol. 136 (2007) 193.

[11] Y. Zhang, J. H. Hao, C. L. Mak, X. H. Wei, Opt. Express 19 (2012) 1824.

[12] M. Saif, J. Photochem. Photobiol. A-Chem. 205 (2009) 145.

[13] M. A. Meneses-Nava, O. Barbosa-Garcia, J. L. Maldonado, G. Ramos-Ortiz, J. L. Pichardo, M. TorresCisneros, M. Garcia-Hernandez, A. Garcia-Murillo, F. J. Carrillo-Romo, Opt. Mater. 31 (2008) 252.

[14] I. K. Battisha, J. Sol-Gel Sci. Technol. 30 (2004) 163.

[15] M. Mohapatra, Y. P. Naik, V. Natarajan, T. K. Seshagiri, S. V. Godbole, Physica B 406 (2011) 1977.

[16] R. Reshmi, R. Sreeja, M. K. Jayaraj, J. James, M. T. Sebastian, Appl. Phys. B-Lasers Opt. 96 (2009) 433.

[17] L. S. Cavalcante, A. Z. Simoes, J. W. M. Espinosa, L. P. S. Santos, E. Longo, J. A. Varela, P. S. Pizani, J. Alloy. Compd. 464 (2008) 340.

[18] W. F. Zhang, J. W. Tang, J. H. Ye, Chem. Phys. Lett. 418 (2006) 174.

[19] P. T. Diallo, P. Boutinaud, R. Mahiou, J. C. Cousseins, Phys. Status Solidi A-Appl. Res. 160 (1997) 255.

[20] R. Chen, F. L. Song, D. H. Chen, Y. H. Peng, Powder Technol. 194 (2009) 252.

[21] R. Pazik, R. J. Wiglusz, W. Strek, Mater. Res. Bull. 44 (2009) 1328.

[22] P. Boutinaud, E. Tomasella, A. Ennajdaoui, R. Mahiou, Thin Solid Films 515 (2006) 2316.

[23] X. H. Pan, J. D. Yu, Y. Liu, S. Yoda, H. M. Yu, M. H. Zhang, F. Ai, F. Jin, W. Q. Jin, J. Alloy. Compd. 509 (2011) 7504 .

[24] C. Peng, Z. Y. Hou, C. M. Zhang, G. G. Li, H. Z. Lian, Z. Y. Cheng, J. Lin, Opt. Express 18 (2010) 7543.

[25] T. M. Mazzo, M. L. Moreira, I. M. Pinatti, F. C. Picon, E. R. Leite, I. L. V. Rosa, J. A. Varela, L. A. Perazolli, E. Longo, Opt. Mater. 32 (2010) 990.

[26] L. L. Noto, S. S. Pitale, M. A. Gusowki, J. J. Terblans, O. M. Ntwaeaborwa, H. C. Swart, Powder Technol. 237 (2013) 141.

[27] P. Bohacek, S. Zazubovich, N. Solovieva, M. Nikl, Opt. Mater. 30 (2007) 66.

[28] T. M. H. Duong, N. H. Nam, L. V. Vu, N. N. Long, J. Alloy. Compd. 537 (2012) 54.

[29] J. P. Fu, Q. H. Zhang, Y. G. Li, H. Z. Wang, J. Alloy. Compd. 485 (2009) 418.

[30] A. T. de Figueiredo, S. de Lazaro, E. Longo, E. C. Paris, J. A. Varela, M. R. Joya, P. S. Pizani, Chem. Mat. 18 (2006) 
2904.

[31] P. R. de Lucena, E. R. Leite, F. M. Pontes, E. Longo, P. S. Pizani, J. A. Varela, J. Solid State Chem. 179 (2006) 3997. [32] F. V. Motta, A. T. de Figueiredo, V. M. Longo, V. R. Mastelaro, A. Z. Freitas, L. Gomes, N. D. Vieira, E. Longo, J. A. Varela, J. Lumin. 129 (2009) 686.

[33] W. H. Zhang, J. X. Zhang, J. Lumin. 131 (2011) 2307. [34] V. A. Pustovarov, T. V. Perevalov, V. A. Gritsenko, T. P. Smirnova, A. P. Yelisseyev, Thin Solid Films 519 (2011) 6319.

[35] T. Li, M. R. Shen, L. Fang, F. G. Zheng, X. L. Wu, J. Alloy. Compd. 474 (2009) 330.

[36] E. R. Vance, R. A. Day, Z. Zhang, B. D. Begg, C. J. Ball, M. G. Blackford, J. Solid State Chem. 124 (1996) 77.

[37] I. Sabikoglu, J. Alloy. Compd. 556 (2013) 135.

[38] W. H. Zhang, H. Y. Wang, J. Chen, W. H. Zhang, X. M. Xiong, J. X. Zhang, J. Lumin. 128 (2008) 1359.

[39] E. Pinel, P. Boutinaud, G. Bertrand, C. Caperaa, J. Cellier, R. Mahiou, J. Alloy. Compd. 374 (2004) 202.

[40] G. F. Teixeira, M. A. Zaghete, G. Gasparotto, M. G. S. Costa, J. W. M. Espinosa, E. Longo, J. A. Varela, J. Alloy. Compd. 512 (2012) 124.

[41] F. C. D. Lemos, D. M. A. Melo, J. E. C. da Silva, Opt. Comm. 231 (2004) 251.

[42] G. Gasparotto, S. A. M. Lima, M. R. Davolos, J. A.
Varela, E. Longo, M. A. Zaghete, J. Lumin. 128 (2008) 1606. [43] Y. X. Pan, Q. Su, H. F. Xu, T. H. Chen, W. K. Ge, C. L. Yang, M. M. Wu, J. Solid State Chem. 174 (2003) 69.

[44] A. E. Souza, G. T. A. Santos, R. A. Silva, M. L. Moreira, D. P. Volanti, E. C. Paris, S. R. Teixeira, E. Longo, Int. J. Appl. Ceram. Technol. 9 (2011) 186.

[45] S. Qin, A. I. Becerro, F. Seifert, J. Gottsmann, J. Z. Jiang, J. Mater. Chem. 10 (2000) 2401.

[46] S. Qin, A. I. Becerro, F. Seifert, J. Gottsmann, J. Z. Jiang, J. Mater. Chem. 10 (2000) 1609.

[47] S. Qin, X. Wu, F. Seifert, A. I. Becerro, J. Chem. Soc.Dalton Trans. (2002) 3751.

[48] B. D. Cullity, Elements of X-ray diffraction, AddisonWesley Publ. Co., Reading (1978).

[49] C. A. Kodaira, H. F. Brito, E. E. S. Teotonio, M. Claudia, M. Felinto, O. L. Malta, G. E. S. Brito, J. Braz. Chem. Soc. 15 (2004) 890.

[50] T. Honma, Y. Benino, T. Fujiwara, R. Sato, T. Komatsu, J. Phys. Chem. Solids 65 (2004) 1705.

[51] P. T. Diallo, K. Jeanlouis, P. Boutinaud, R. Mahiou, J. C. Cousseins, J. Alloy. Compd. 323 (2001) 218.

[52] N. Suriyamurthy, B. S. Panigrahi, J. Rare Earths 28 (2010) 488.

[53] F. A. Kroger, H. J. Vink, Solid State Phys. 3 (1956) 307. (Rec. 11/06/2013, Ac. 12/10/2013) 